\title{
Blitzphänomene im österreichischen Hoch- und Höchstspannungsnetz - transient erfasst
}

\author{
S. Pack OVE, J. Plesch, L. Schwalt OVE
}

\begin{abstract}
In den letzten Jahren konnten vermehrt transiente Messungen im österreichischen Hochspannungssystem durchgeführt werden. Basierend auf diesen Messungen und den aufgezeichneten Spannungen war es möglich, Signalsequenzen auszuwerten, welche auf direkte atmosphärische Entladungen sowie auf die Auswirkungen von Kopplungseffekten bei ins Erdreich abgeleiteten Blitzentladungen zurückzuführen sind. Zur Erfassung dieser transienten Spannungen werden breitbandige ohmsch-kapazitive Spannungsteiler in allen Spannungsebenen eingesetzt. Eine Korrelation zwischen den gemessenen transienten Spannungen und den Daten des österreichischen Blitzortungssystems ALDIS Austrian Lightning Detection and Information System wurde im Zuge der Untersuchungen durchgeführt. In diesem Beitrag werden drei typische transiente Ereignisse, verursacht durch Blitzentladungen, vorgestellt.
\end{abstract}

Schlüsselwörter: atmosphärische Entladungen; transiente Messungen; Blitzortungssystem; Hoch- und Höchstspannungsnetz

Lightning phenomena in the Austrian transmission system-transiently recorded.

In the last years transient measurements at several substations in the Austrian transmission system have been performed. Based on these measurements it was possible to detect signal sequences which correlate with direct and nearby lightning discharges as well as the coupling effect to phases after lightning strikes to the ground wire. These transient voltages were measured by using resistivecapacitive voltage dividers (RC-divider) to generate a high quality dataset. The high bandwidth of the RC-divider and its resulting frequency stability is needed to analyze such transient events. A correlation between the measurements and the data of the Austrian Lightning Detection and Information System (ALDIS) was performed. In this paper, three different representative measurements of transients caused by atmospheric discharges to or close to transmission lines will be presented.

Keywords: atmospheric discharges; transient measurement; lightning location system; high voltage transmission system

Eingegangen am 6. Juli 2017, angenommen am 24. August 2017, online publiziert am 22. November 2017

(c) The Author(s) 2017. Dieser Artikel ist auf Springerlink.com mit Open Access verfügbar

\section{Einleitung}

Die Erfassung transienter Vorgänge in Hochspannungssystemen hat in den letzten Jahren wieder an großer Bedeutung gewonnen. Atmosphärische Entladungen auf bzw. neben Hochspannungsfreileitungen führen bekanntlich zu überlagerten transienten Spannungen in den Systemen und haben Auswirkungen auf die Isolationskoordination [1]. Solche Einwirkungen äußern sich durch Spannungseinbrüche oder Spannungsspitzen und wirken sich direkt auf die Spannungsqualität und damit auf die Systemperformance der Hochspannungssysteme aus. Für die grundlegende Analyse von Einwirkungen atmosphärischer Entladungen auf Hochspannungsfreileitungen und Systeme werden prinzipiell numerische Berechnungsprogramme herangezogen. Dennoch ist es unumgänglich, mit moderner Messtechnik diese transienten Einwirkungen zu erfassen, um die nach Theorie durchgeführten Analysen zu bestätigen. Auch Einschläge im nahen Umfeld einer Hochspannungsleitung äußern sich durch transiente Einkopplungen [2].

Um hochqualitative Messdaten solcher transienten Ereignisse im österreichischen Übertragungsnetz erfassen zu können, wurden in ausgewählten Knotenpunkten von Netzbetreibern breitbandige ohmsch-kapazitive Spannungsteiler (RC-Teiler) installiert. Die Analyse von geplanten Schalthandlungen und ungeplanten Fehlerzuständen kann mit Hilfe der internen Betriebsaufzeichnungen korreliert werden. Eine Analyse von Messsequenzen hinsichtlich atmosphä- rischen Entladungen kann mit Ortungsdaten des österreichischen Blitzortungssystems ALDIS verglichen werden.

Diese Arbeit beschäftigt sich mit den Spannungsverläufen von direkten und indirekten atmosphärischen Entladungen auf Hochspannungsfreileitungen. Um den Eintrag dieser transienten Effekte zu demonstrieren, wurden drei signifikante Messsequenzen aus den umfangreichen Datensätzen ausgewählt. Das erste Fallbeispiel zeigt eine atmosphärische Entladung als direkten Einschlag in ein Hochspannungssystem. Die beiden weiteren Fallbeispiele zeigen den Ablauf von indirekten Einkopplungen und Spannungsüberlagerungen zur netzfrequenten Hochspannung, welche durch Blitzentladungen in der Umgebung einer Freileitung verursacht wurden.

2. Atmosphärische Entladungen als transiente Störquellen Das Gebiet der transienten Abläufe wird in Frequenzbereiche unterteilt. Für die durchgeführten Messungen wurden grundsätzlich

Pack, Stephan, Institut für Hochspannungstechnik und Systemmanagement, Technische Universität Graz, Inffeldgasse 18, 8010 Graz, Österreich (E-Mail: pack@tugraz.at);

Plesch, Jürgen, Institut für Hochspannungstechnik und Systemmanagement, Technische Universität Graz, Inffeldgasse 18, 8010 Graz, Österreich

(E-Mail: juergen.plesch@tugraz.at); Schwalt, Lukas, Institut für Hochspannungstechnik und Systemmanagement, Technische Universität Graz, Inffeldgasse 18, 8010 Graz, Österreich (E-Mail: lukas.schwalt@tugraz.at) 


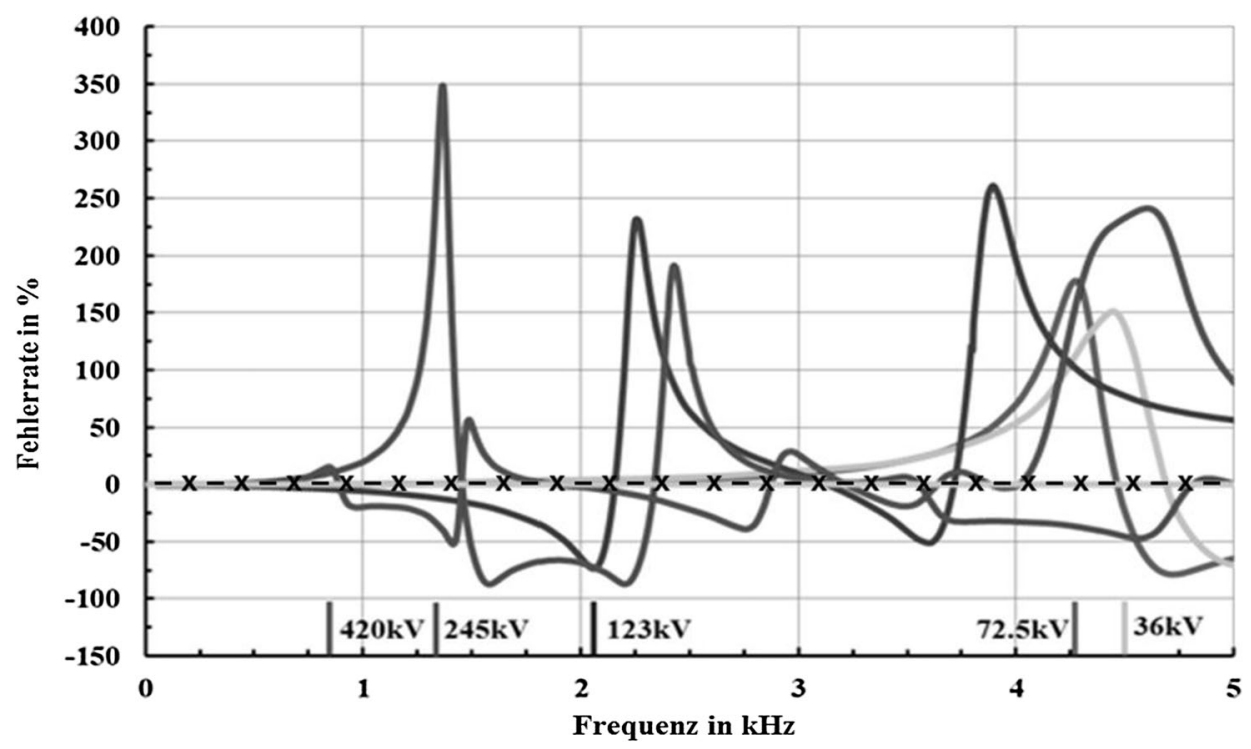

Abb. 1. Übertragungsverhalten induktiver Messwandler bis $5 \mathrm{kHz}$ und Vergleich mit dem RC-Teiler. Fehlerrate in Prozent für einen induktiven 36-kV-, 72,5-kV-, 123-kV-, 245-kV-, 420-kV-Messwandler (Graustufen) und des ohmsch-kapazitiven 420-kV-Spannungsteilers (-x -) [3]

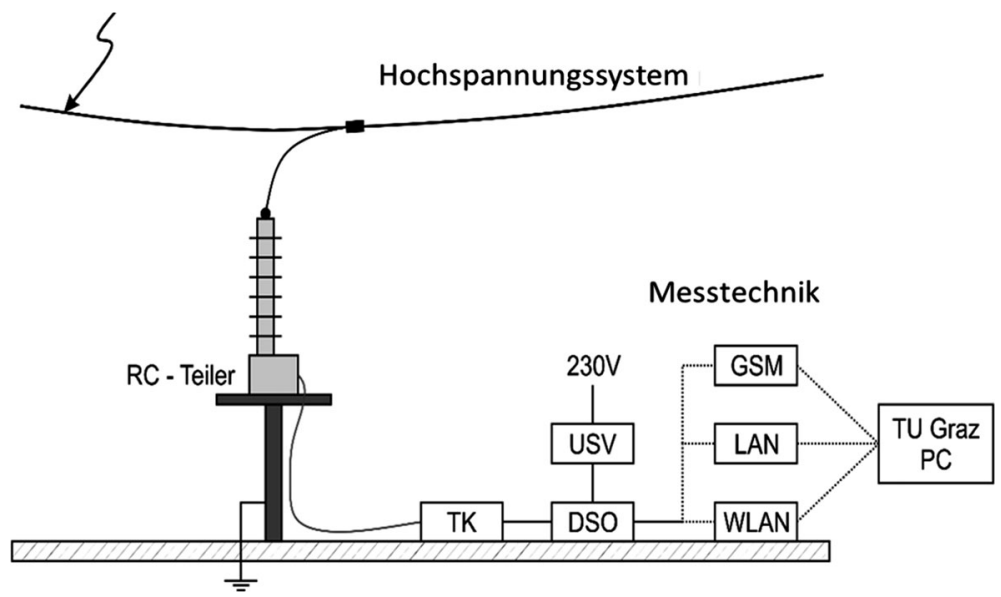

Abb. 2. Schematische Darstellung des transienten Messaufbaus. Messtechnik: Tastkopfsystem (TK), Transientenrecorder (DSO), Unterbrechungsfreie Stromversorgung (USV), Remote Steuerung über GSM, LAN oder WLAN

Signale mit Frequenzen von mehreren hundert $\mathrm{Hz}$ bis einigen $\mathrm{MHz}$ betrachtet. Atmosphärische Entladungen laufen in einem Frequenzbereich von $\mathrm{kHz}$ bis $\mathrm{MHz}$ ab. Vor allem um diese schnell ablaufenden Phänomene sichtbar zu machen, gilt es, die installierten Schutz- und Überwachungssysteme der Netzbetreiber zu erweitern. Die gängigen Systeme der Hochspannungsanlagen sind für die Aufbereitung von Signalen im 50-Hz-Bereich ausgelegt und übermitteln im transienten Bereich nur ungenau bzw. verfälscht die ablaufenden Vorgänge [3]. Abbildung 1 zeigt einen Vergleich des Übertragungsverhaltens verschiedener induktiver Messwandler bis $5 \mathrm{kHz}$ und eines RCTeilers. Daraus kann entnommen werden, dass bei Verwendung eines induktiven Messwandlers in diesem Frequenzbereich Fehler von bis zu 350\%, abhängig von der Spannungsebene, auftreten können.

Um die genannten transienten Ereignisse und im speziellen die atmosphärischen Entladungen in hoher Qualität erfassen zu können, bedarf es des Einsatzes von RC-Teilern, welche über einen großen Bereich ein lineares und reproduzierbares Übertragungsverhalten aufweisen.

\section{Messmethodik Blitzphänomene}

Um die Auswirkung von atmosphärischen Entladungen im Hochspannungssystem sichtbar zu machen, bedarf es eines Messaufbaus im Hochspannungssystem zur Erfassung transienter, höherfrequenter Spannungen sowie der zeitgenauen Ortungsdaten von ALDIS (siehe [4] und [5]).

3.1 Transiente Messung von Blitzentladungen mit RC-Teilern Ohmsch-kapazitive Spannungsteiler werden heute als modernes Messinstrument in Hochspannungssystemen für die Erfassung transienter Spannungen eingesetzt. Für die Konzeptionierung eines Messaufbaus in Hochspannungsanlagen wird neben dem RC-Teiler ein transienten-tauglicher Messtechnikkreis benötigt (Abb. 2).

Mit dem Einsatz von RC-Teilern in einer Hochspannungsanlage kann das transiente Verhalten bei atmosphärischen Entladungen 
untersucht werden. Hierfür erfolgte die Installation von einem RCTeiler je Phase, meist im Abspannfeld einer ausgewählten Hochspannungsfreileitung oder an geeigneter Stelle im Umspannwerk.

Der Grund für den zusätzlichen Aufwand des Einbaus von RCTeilern in Hochspannungsanlagen ist in der hochqualitativen Erfassung der Leiter-Erde-Spannung über eine definierte Bandbreite begründet (siehe [3] und [6]). In Kombination mit einem TransientenMesssystem können die gemessenen Spannungen dauernd aufgezeichnet werden. Mit den gefilterten und ausgewerteten transienten Spannungsverläufen aus den Messungen und den Informationen des Blitzortungssystems kann eine Korrelation hergestellt werden, um die Einwirkungen von direkten und nahen Einschlägen auf die Spannungsqualität zu evaluieren.

\subsection{Blitzortungssystem}

ALDIS betreibt seit 1991 ein über Österreich verteiltes Netzwerk aus acht Blitzortungssensoren. Im Jahr 2001 wurde ALDIS einer der Hauptoperatoren des europäischen Blitzortungsnetzwerks EUCLID und hat somit Zugriff auf über 150 Sensoren in Europa [7]. Die Ortungsgenauigkeit ist ein wesentlicher Parameter, um eine Korrelation mit den transienten Messergebnissen in Hochspannungssystemen durchführen zu können. Durch ständige Anpassung und Verbesserung des Systems konnte in den letzten Jahren der mittlere Ortungsfehler auf $100 \mathrm{~m}$ verringert werden [8].

Zur Verbesserung der Ortungsgenauigkeit kann ein Abgleich der georteten Entladungen mit Turmeinschlägen oder tatsächlich gemessenen Bodeneinschlägen (sogenannten "Ground Truth Daten") erfolgen. Neben den bekannten Arbeiten am Gaisberg Tower bei Salzburg [9] wird daher am Institut für Hochspannungstechnik und Systemmanagement der TU Graz auf diesem Gebiet ein Forschungsprojekt "Lightning Observation in the Alps (LiOn)" betrieben, bei dem in alpinen Regionen Hochgeschwindigkeitsvideo- und Feldmessdaten erfasst und ausgewertet werden.

\section{Korrelation transienter Messung mit Blitzortungsdaten}

Eine Korrelation dieser Art wurde bereits im 110-kV-Hochspannungsnetz unter Verwendung von Blitzortungsdaten durchgeführt [10]. Nach Stand der Technik werden in Österreich derzeit für die Spannungsmessung im Hoch- und Höchstspannungsnetz induktive Messwandler eingesetzt. Die Frequenzcharakteristik solcher induktiven Messwandler erlaubt es nicht, über den Frequenzbereich ein eindeutiges Abbild höherfrequenter transienter Abläufe zu liefern. Diese sind zur 50-Hz-Erfassung für Regel- und Schutzzwecke ausgelegt, nicht aber für die Erfassung höherfrequenter, transienter Signale.

Die Stördatenerfassung in einem Netz wird im Normalfall nur bei einer Leitungsabschaltung gestartet. Mit der Installation von RCTeilern und einem transienten Messsystem wird es möglich, jegliche Art von transienten Spannungssignalen, wie z.B. auch induzierte Spannungen ausgelöst durch atmosphärische Entladungen in der Umgebung oder durch direkte Einschläge ins Erdseil, zu erfassen, und in weiterer Folge in hoher Genauigkeit zu identifizieren und zu analysieren.

\subsection{Herangezogene Datensätze}

Für die Auswertung der vorgestellten transienten Messungen wurde ein Blitzortungsdatensatz für den gesamtem Messzeitraum erstellt. Mit Hilfe der Geoinformationssoftware QGIS konnte mit einem eigens entwickelten Tool die betrachtete Region eingegrenzt werden, um für die projektspezifischen Anforderungen einen zugeordneten Datensatz der atmosphärischen Entladungen zu generieren.
Mit dem transienten Messsystem werden Leiter-Erde-Spannungen über ein definiertes Zeitfenster aufgezeichnet. Abhängig vom Untersuchungsgegenstand, Langzeitaufzeichnung oder Aufzeichnung von Blitzphänomenen, ist es erforderlich, mittels Fernzugriff das Messgerät an die aktuell vorliegende Situation eines Hochspannungsnetzes anzupassen. Durch die automatisierte triggerbasierte Auslösung wird innerhalb eines Messzeitraums ein großer Datensatz generiert, welcher zeitgerecht durch Fernzugriff abgeholt und gesichtet werden muss

Aus der Vielzahl der aufgezeichneten Messsequenzen ist es in vielen Fällen nur eingeschränkt möglich, dass direkt aus dem Spannungsverlauf auf die Ursache geschlossen werden kann. Vor allem setzt eine solche Zuweisung Expertenwissen voraus. Mit der Kombination wissenschaftlicher Teilgebiete können aber zuvor nicht zuordenbare Messsequenzen eindeutig zugeordnet werden.

Durch die Korrelation der aufgezeichneten transienten Spannungssequenzen mit den internen Aufzeichnungen der Netzbetreiber kann ein Teil der Signalverläufe geplanten Schalthandlungen im betrachteten Systembereich oder umliegenden Umspannwerken zugeordnet werden. Auch unvorhersehbare transiente Signale werden, sofern eine direkte Auswirkung auf das Hochspannungssystem eintritt, protokolliert. Der verbleibende Datensatz wurde für diese Publikation mit dem generierten Datensatz der georteten atmosphärischen Entladungen korreliert.

\subsection{Analyseverfahren}

Für die Auswertung und den Vergleich der transienten Messung mit den Blitzortungsdaten wurde ein Analyseprogramm entwickelt. Die grundlegenden Eingangsparameter umfassen die Daten des Blitzortungssystems, die transienten Messungen und die geographischen Daten einer Hochspannungsfreileitung.

Bisher wurden atmosphärische Entladungen, welche in einem Korridor vom $\pm 1 \mathrm{~km}$ der Hochspannungsfreileitung detektiert wurden, als Störquellen, welche Leitungsabschaltungen usw. zur Folge haben, angesehen [11]. Für diese Analyse wurde der Korridorbereich hinsichtlich induzierter Überspannungen bei atmosphärischen Entladungen ins Erdreich vergrößert (Korridorbreite bis zu $5 \mathrm{~km}$ um das System) und die Analysemethodik (Software) dahingehend angepasst.

Als Identifikationsmerkmal eines Hauptblitzes mit folgenden Teilblitzen wurde die relative zeitliche Differenz zwischen diesen Ereignissen gewählt. Dadurch wird eine hochgenaue Zuordnung von atmosphärischen Entladungen zu den transient aufgezeichneten Messungen gewährleistet.

\section{Fallbeispiele}

Im folgenden Abschnitt werden drei Beispiele von transienten Messungen im Hochspannungssystem ausgewählt, welche die unterschiedlichen Auswirkungen auf den Netzspannungsverlauf aufzeigen.

\subsection{Direkter Blitzschlag}

Im ersten Fallbeispiel konnte eine atmosphärische Entladung als direkter Einschlag in eine Hochspannungsfreileitung identifiziert werden.

Aus den Ortungsdaten kann ein Abstand des Einschlags zur Hochspannungsleitung von 0,09 km bzw. 1,91 km für Haupt- bzw. Folgeblitz abgelesen werden. Die Ortungsdaten der atmosphärischen Entladung sind in Tab. 1 zusammengefasst.

Für den Hauptblitz wurde eine Blitzstromamplitude von -11,05 kA berechnet. Dieser verursachte auf Phase L2 einen einpoligen Spannungszusammenbruch (siehe Abb. 3). 


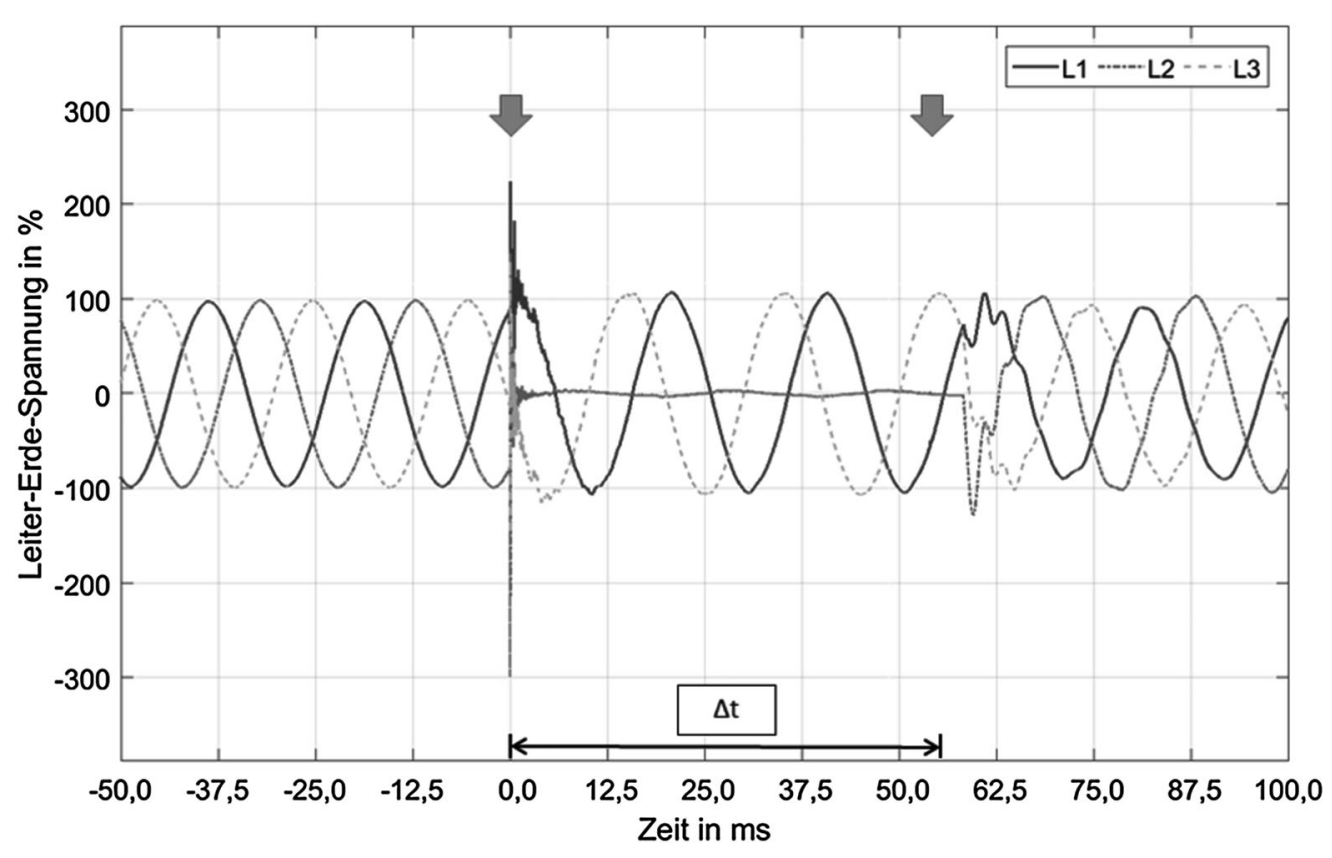

Abb. 3. Fallbeispiel 1: Transiente Spannungsverläufe der drei Phasen im Hochspannungssystem und Zeitabstand zwischen Haupt- und Folgeblitz $(\Delta t)$

Tab. 1. Blitzortungsdaten für Fallbeispiel 1 (30.06.2016). Abkürzungen: Amp = Blitzstromamplitude in $\mathrm{kA}$, $\mathrm{ns}=$ Anzahl der Teilbitze, sn $=$ Teilblitznummer, Dist $=$ Distanz des georteten Einschlags zum Hochspannungssystem in $\mathrm{km}, \Delta t=$ Zeitdifferenz zum vorangegangenen Teilblitz in $\mathrm{ms}$

\begin{tabular}{lllllll}
\hline $\begin{array}{l}\text { Uhrzeit } \\
\text { (hh:mm:ss) }\end{array}$ & $\begin{array}{l}\text { Nanosec } \\
\text { (ns) }\end{array}$ & $\begin{array}{l}\text { Amp } \\
\text { (kA) }\end{array}$ & $\begin{array}{l}\text { ns } \\
(-)\end{array}$ & $\begin{array}{l}\text { sn } \\
(-)\end{array}$ & $\begin{array}{l}\text { Dist } \\
(\mathrm{km})\end{array}$ & $\begin{array}{l}\Delta t \\
(\mathrm{~ms})\end{array}$ \\
\hline $20: 11: 25$ & 126461440 & $-11,05$ & 2 & 1 & 0,09 & \\
20:11:25 & 180478720 & $-8,1$ & 2 & 1 & 1,91 & 54,01 \\
\hline
\end{tabular}

Nach einer Zeitdauer von 54,01 ms kann der Folgeblitz durch eine zusätzlich in allen drei Phasen induzierte Spannung detektiert werden. Die Spannungsstabilisierung nahm insgesamt $250 \mathrm{~ms}$ in Anspruch.

5.2 Induzierte Überspannung - Korrelation Blitzortung Das zweite Fallbeispiel zeigt eine negative Wolke-Erde-Entladung bestehend aus einem Haupt- und drei Folgeblitzen. Die Blitze wurden in einer Distanz von 0,09 km bis 1,09 km zur Freileitung geortet. Die Daten des Blitzortungssystems sind in Tab. 2 angeführt. Der Hauptblitz hatte eine Blitzstromstärke von -99,04 kA und verursachte keinen Leitungsausfall (siehe Abb. 4). Durch die in alle drei Phasen gleich ablaufende Spannungsanhebung zu den Zeitpunkten der atmosphärischen Entladungen kann von einer induzierten Überspannung ausgegangen werden. Mit einer maximalen Überspannung von ca. $40 \%$ auf Phase L3 wurde die Spannungsbeanspruchung des Systems nicht überschritten, weshalb es zu keiner Schutzauslösung kam.

5.3 Induzierte Überspannung - Signalanalyse

Das dritte Fallbeispiel zeigt induzierte Spannungen, welche von einer atmosphärischen Entladung, bestehend aus einem Haupt und zwei Folgeblitzen, hervorgerufen wurden. Die drei Teilblitze hatten
Tab. 2. Blitzortungsdaten für Fallbeispiel 2 (30.06.2016). Abkürzungen: $A m p=$ Blitzstromamplitude in $\mathrm{kA}$, $\mathrm{ns}=$ Anzahl der Teilbitze, sn $=$ Teilblitznummer, Dist $=$ Distanz des georteten Einschlags zum Hochspannungssystem in $\mathrm{km}, \Delta t=$ Zeitdifferenz zum vorangegangenen Teilblitz in ms

\begin{tabular}{lllllll}
\hline $\begin{array}{l}\text { Uhrzeit } \\
\text { (hh:mm:ss) }\end{array}$ & $\begin{array}{l}\text { Nanosec } \\
\text { (ns) }\end{array}$ & $\begin{array}{l}\text { Amp } \\
\text { (kA) }\end{array}$ & $\begin{array}{l}\mathrm{ns} \\
(-)\end{array}$ & $\begin{array}{l}\text { sn } \\
(-)\end{array}$ & $\begin{array}{l}\text { Dist } \\
(\mathrm{km})\end{array}$ & $\begin{array}{l}\Delta t \\
(\mathrm{~ms})\end{array}$ \\
\hline $20: 17: 37$ & 79693568 & $-99,03$ & 4 & 1 & 1,09 & \\
$20: 17: 37$ & 99759104 & $-17,3$ & 4 & 2 & 0,23 & 20,06 \\
$20: 17: 37$ & 122703360 & $-29,54$ & 4 & 3 & 0,09 & 22,94 \\
$20: 17: 37$ & 157830656 & $-21,32$ & 4 & 4 & 0,26 & 35,12 \\
\hline
\end{tabular}

Tab. 3. Blitzortungsdaten für Fallbeispiel 3 (02.07.2015). Abkürzungen: $A m p=$ Blitzstromamplitude in $k A, n s=$ Anzahl der Teilbitze, sn $=$ Teilblitznummer, Dist $=$ Distanz des georteten Einschlags zum Hochspannungssystem in $\mathrm{km}, \Delta t=$ Zeitdifferenz zum vorangegangenen Teilblitz in ms

\begin{tabular}{lllllll}
\hline $\begin{array}{l}\text { Uhrzeit } \\
\text { (hh:mm:ss) }\end{array}$ & $\begin{array}{l}\text { Nanosec } \\
\text { (ns) }\end{array}$ & $\begin{array}{l}\text { Amp } \\
(\mathrm{kA})\end{array}$ & $\begin{array}{l}\mathrm{ns} \\
(-)\end{array}$ & $\begin{array}{l}\text { sn } \\
(-)\end{array}$ & $\begin{array}{l}\text { Dist } \\
(\mathrm{km})\end{array}$ & $\begin{array}{l}\Delta t \\
(\mathrm{~ms})\end{array}$ \\
\hline 15:46:45 & 399124992 & $-5,89$ & 3 & 1 & 1,74 & \\
$15: 46: 45$ & 445764864 & $-7,52$ & 3 & 2 & 1,51 & 46,6 \\
$15: 46: 45$ & 555971328 & $-45,2$ & 3 & 3 & 1,59 & 110,2 \\
\hline
\end{tabular}

eine kalkulierte Distanz zur Freileitung von 1,51 bis 1,74 km. Die Daten des Blitzortungssystems sind in Tab. 3 angeführt. Der Hauptblitz induziert auf allen drei Phasen eine Überspannung von ca. 5\% (Blitzstromstärke -5,89. Siehe Abb. 5, Bildfolge 1). Die Folgeentladungen Nummer 2 und 3 induzierten Überspannungen von ca. 10\% (-7,52 kA) bzw. 25\% (-45,2 kA) (siehe Abb. 5, Bildfolge 2 bzw. 3). Die Spannungsanhebung tritt in allen drei Phasen gleich auf (siehe 


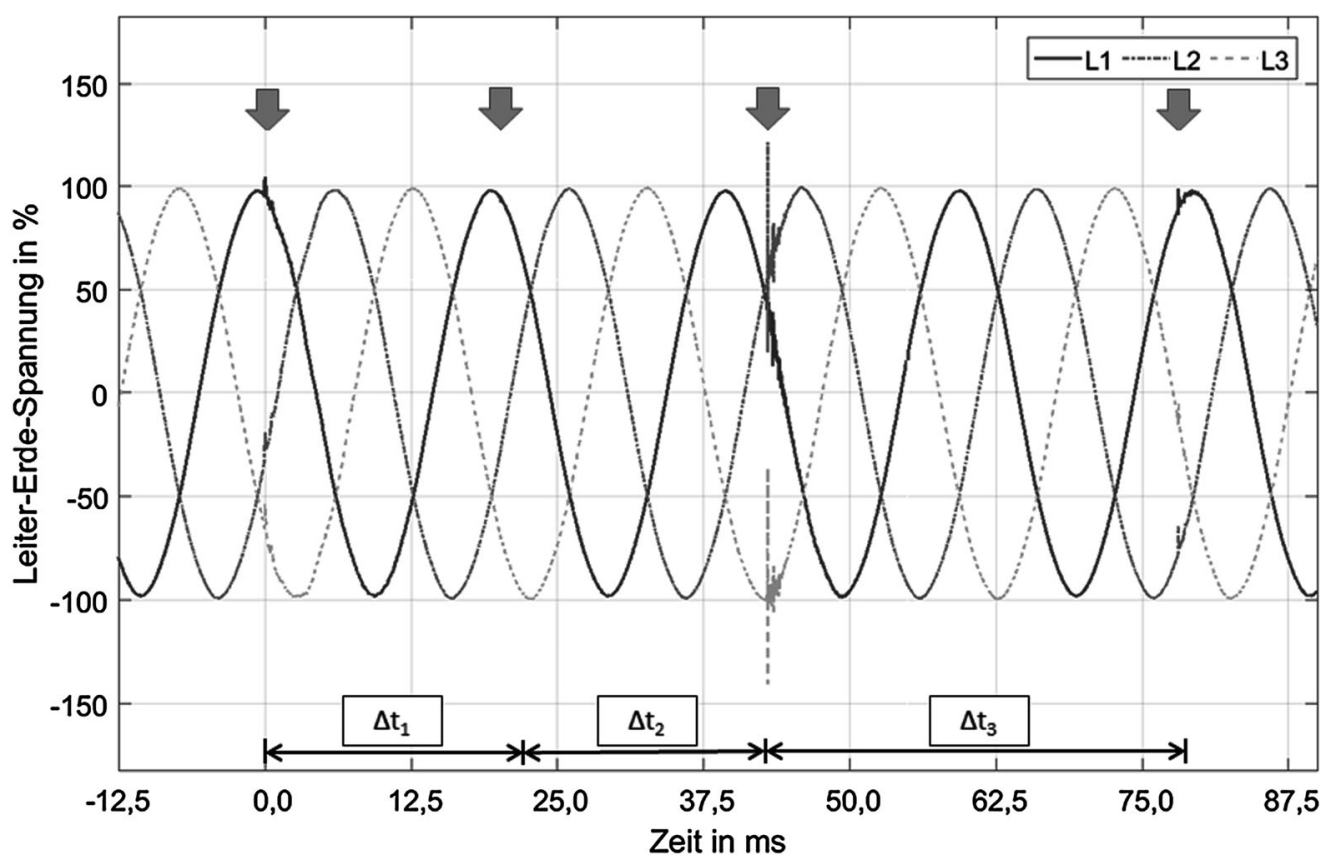

Abb. 4. Fallbeispiel 2: Transiente Spannungsverläufe der drei Phasen im Hochspannungssystem und Zeitabstand zwischen Haupt- und Folgeblitz $\left(\Delta t_{x}\right)$

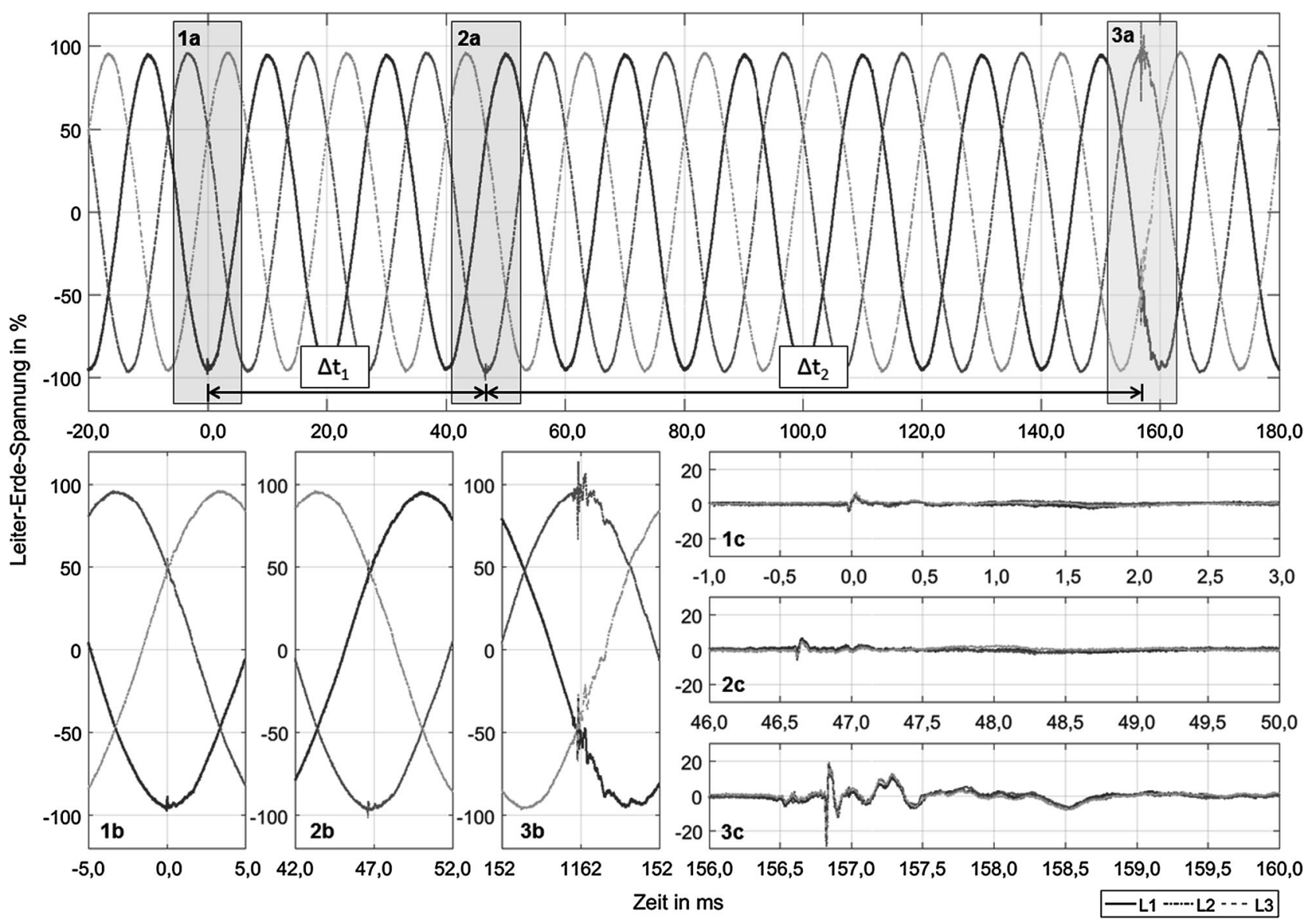

Abb. 5. Fallbeispiel 3: Transiente Spannungsverläufe und Zeitabstände zwischen Haupt- und Folgeblitze $\left(\Delta t_{x}\right)(B i l d f o l g e ~ a)$. Zoomfenster der Spannungsverläufe zum Zeitpunkt der Überspannung (Bildfolge $b$ ). Spannungsverläufe der drei Phasen zum Zeitpunkt der Überspannung bei herausgerechneter Grundschwingung (Bildfolge c) 
Abb. 5, Bildfolge c), weshalb von einer induzierten Überspannung ausgegangen werden kann.

\section{Zusammenfassung und Ausblick}

Diese Arbeit gibt einen Einblick in die Forschungsaktivitäten am Institut für Hochspannungstechnik und Systemmanagement der TU Graz auf dem Gebiet Messung, Erfassung und Analyse transienter Phänomene in Hochspannungsnetzen, die in Kooperation mit Netzbetreibern und ALDIS durchgeführt werden. Durch die moderne Messtechnik mit RC-Teilern und digitalen Transienten-Recordern können transiente Spannungen in allen Spannungsebenen mit einer messtechnischen Gesamtqualität aufgezeichnet werden, wie es bisher nicht möglich war.

Die Erfassung transienter Vorgänge in Hochspannungssystemen mit hoher Zeitgenauigkeit und hoher transienter Messqualität hat in den letzten Jahren wieder an Bedeutung gewonnen. Die beschriebenen Spannungsmessungen können einerseits die Auswirkungen von direkten Einschlägen in die Leitungen und andererseits die induzierenden Wirkungen von atmosphärischen Entladungen auf den Spannungsverlauf in Hochspannungssystemen aller Spannungsebenen aufzeigen. Durch die Korrelation dieser Daten mit Daten des Blitzortungssystems können den auftretenden Spannungsabweichungen oder Überspannungen bestimmte Haupt- bzw. Folgeentladungen als Ursache eindeutig zugewiesen werden. Die zeitliche Genauigkeit der transienten Messdaten und der Blitzortungsdaten im Nanosekundenbereich macht diese Zuordnung unter Zuhilfenahme der relativen Zeitabstände zwischen Haupt- und Folgeentladung bzw. zwischen einzelnen Folgeentladungen erst möglich.

In drei Fallbeispielen konnten die transienten Spannungsverläufe dargestellt und analysiert werden. Dabei konnten die unterschiedlichen transienten Auswirkungen durch einen direkten Blitzeinschlag in eine Freileitung, durch einen Erdseileinschlag und durch induzierte Spannungen auf den Netzspannungsverlauf nachgewiesen werden.

In Rahmen dieser Forschungsaktivitäten werden auch eine Vielzahl anderer Auswirkungen auf das Hochspannungsnetz sowie anderer atmosphärischer Entladungen korreliert und analysiert. Dazu werden am Institut in Kooperation mit ALDIS und der ZAMG und dem Übertragungsnetzbetreiber APG, seit einigen Jahren in einem neuen Forschungsprojekt "Lightning Observation in the Alps (LiOn)" Vor-Ort-Messungen von atmosphärischen Bodeneinschlägen mit einem Hochgeschwindigkeitsvideo- und Feldmesssystem im österreichischen Alpenraum und in der Nähe von Hochspannungssystemen durchgeführt.

\section{Autoren}

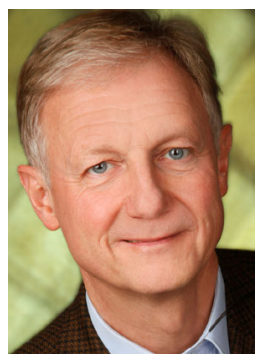

\section{Stephan Pack}

Jahrgang 1958, Studium der Elektrotechnik an der Technischen Universität Graz, Österreich, seit 1985 am Institut für Hochspannungstechnik und Systemmanagement der TU Graz und an der Versuchsanstalt für Hochspannungstechnik Graz GmbH auf den Gebieten transiente Vorgänge in Hochspannungssystemen und Übertragungsnetzen, Isolationskoordination, Blitzschutz, Schutzzonenkonzepte und Erdungsanlagen tätig. Allg. beeid. und gerichtl. zertif. Sachverständiger für Hochspannungstechnik und Blitzschutzwesen, diplomierter Umwelttechniker, stellv. Leiter des Instituts. Mitarbeit in OVE, VDE/ABB, ONC, CIGRE.
Diese Arbeiten zeigen, welche Bedeutung und die Möglichkeiten die Erfassung von transienten Vorgängen in Hochspannungssystemen mit modernen RC-Teilern hat, indem neue Erkenntnisse für das Verständnis um die Spannungsqualität und die Netzsicherheit, aber auch durch die Korrelation mit Blitzortungsdaten abgeleitet werden können.

\section{Danksagung}

Open access funding provided by Graz University of Technology.

Open Access This article is distributed under the terms of the Creative Commons Attribution 4.0 International License (http://creativecommons.org/ licenses/by/4.0/), which permits unrestricted use, distribution, and reproduction in any medium, provided you give appropriate credit to the original author(s) and the source, provide a link to the Creative Commons license, and indicate if changes were made.

\section{Literatur}

1. IEC Standard 60071-1 (2006): Insulation coordination, part 1: definitions, principles and rules

2. Paolone, M., Rachidi, F., Borghetti, A., Nucci, C. A., Rubinstein, M., Rakov, V. A., Uman, M. A. (2009): Lightning electromagnetic field coupling to overhead lines: theory, numerical simulations, and experimental validation. IEEE Trans. Electromagn. Compat., 51(3), 532-547.

3. Plesch, J., Sperling, E., Achleitner, G., Pack, S. (2015): Measurement of transient voltage in a substation. In CIGRE symposium: Across borders - HVDC systems and market integration, Lund, Sweden, report 255

4. Diendorfer, G., Schulz, W. (2003): Ground flash density and lightning exposure of power transmission lines. In Power tech conference proceedings. New York: IEEE Press.

5. Diendorfer, G., Pichler, H., Achleitner, G., Broneder, M. (2014): Lightning caused outages in the Austrian power grid transmission line network. In 2014 international conference on lightening protection, ICLP (S. 152-156). New York: IEEE Press.

6. Plesch, J., Pack, S. (2013): On-site measurement of switching transients in high voltage sytems. In: 11. Höfler's days. Slovenia.

7. Schulz, W., Diendorfer, G., Pedeboy, S., Poelman, D. R. (2016): The European lightning location system EUCLID-part 1: performance analysis and validation. Nat. Hazards Earth Syst. Sci., 16(2), 595-605.

8. Schulz, W., Vergeiner, C., Pichler, H., Diendorfer, G., Pack, S. (2012): Validation of the Austrian lightning location system ALDIS for negative flashes. In CIGRE C4 colloquium, Sarajevo, Bosnia and Herzegovina.

9. Diendorfer, G., Pichler, H., Schulz, W. (2014): EUCLID located strokes to the Gaisberg tower-accuracy of location and its assigned confidence ellipse. In Proceedings of international lightning detection conference, ILDC, Tucson, United-States.

10. Plesch, J., Pack, S., Schichler, U., Wurzer, G. (2016): Measurement of transient voltages on a $110 \mathrm{kV}$ high alpine overhead line. In VDE-Hochspannungstechnik 2016 - ETGFachtagung in Berlin, Germany

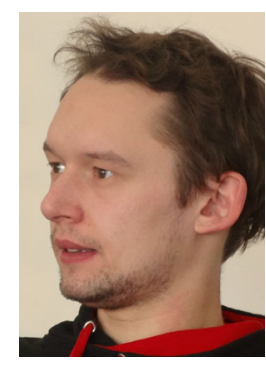

\section{Jürgen Plesch}

Jahrgang 1987, Studium der Elektrotechnik an der Technischen Universität Graz, Österreich, seit 2012 am Institut für Hochspannungstechnik und Systemmanagement der TU Graz auf den Gebieten Isolationskoordination und Überspannungen in Hochspannungssystemen, numerische Berechnungen von Überspannungen, Messung transienter Spannungen und transienter Vorgänge in elektrischen Energiesystemen, Hochspannungsmesstechnik und Resonanzuntersuchungen von Hochspannungsnetzen tätig. 


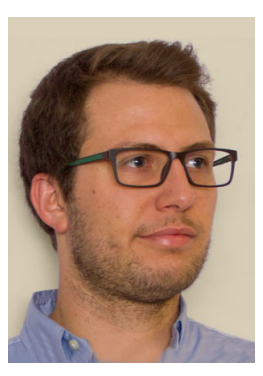

\section{Lukas Schwalt}

Jahrgang 1987, Studium der Elektrotechnik an der Technischen Universität Wien, Österreich, seit 2016 am Institut für Hochspannungstechnik und Systemmanagement der TU Graz auf den Gebieten Wolke-ErdeBlitze im Alpenraum, Messung atmosphärischer Entladungen, Performance von Blitzortungssystemen, Blitzschutzkonzepte, Blitzentladungen in exponierte bauliche Anlagen und transiente Spannungen von Hochspannungsleitungen tätig 